\title{
Cardiac manifestations and diagnostic imaging in pediatric inflammatory multisystem syndrome temporally associated with COVID-19: a systematic review
}

Gilbert Sterling Octavius, ${ }^{1}$ Ricardo Tan, ${ }^{1}$ Teodorus Alfons Pratama, ${ }^{1}$ Charista Lydia Budiputri, ${ }^{1}$ Fellisa Meliani, ${ }^{1}$ Rivaldo Steven Heriyanto, ${ }^{1}$ Rusli Muljadi, ${ }^{2}$ Andry Juliansen ${ }^{3}$

Check for updates

pISSN: 0853-1773 - elSSN: 2252-8083 https://doi.org/10.13181/mji.oa.225754 Med J Indones. 2022;31:20-37

Received: September 06, 2021 Accepted: January 25, 2022

Published online: March 08, 2022

Authors' affiliations:

${ }^{1}$ Faculty of Medicine, Universitas Pelita Harapan, Tangerang, Indonesia, ${ }^{2}$ Department of Radiology, Faculty of Medicine, Universitas Pelita Harapan, Tangerang, Indonesia, ${ }^{3}$ Department of Pediatrics, Faculty of Medicine, Universitas Pelita Harapan, Tangerang, Indonesia

\section{Corresponding author:}

Gilbert Sterling Octavius

Faculty of Medicine, Universitas Pelita Harapan, Tangerang, Indonesia, Jalan Boulevard Jenderal Sudirman, Lippo Karawaci, Tangerang 15811, Indonesia Tel/Fax: +62-21-5460901

E-mail: sterlinggilbert613@hotmail.com

\begin{abstract}
BACKGROUND Several studies have reported pediatric inflammatory multisystem syndrome temporally associated with COVID-19 (PIMS-TS) cases with their cardiac manifestations, but only few studies synthesize the cardiovascular characteristics in children with PIMS-TS. However, detecting cardiac abnormalities is crucial in improving patients' outcomes and reducing mortality. This review aimed to summarize the overall symptoms, laboratory, and workup findings in PIMS-TS patients, focusing on cardiovascular manifestations.
\end{abstract}

METHODS We searched 4 medical databases (PubMed, Science Direct, Medline, and Scielo) and 4 preprint databases (Medrxiv, Research Square, SSRN, and Biorxiv). The literature search was done on November 8, 2021. All case reports, case series, crosssectional studies, cohort studies, and possible clinical trials published from December 2020 onward that studied PIMS-TS on cardiac manifestation (aged 0-18 years) were included. Studies on multisystem inflammatory syndrome in children, animal studies, and studies without full-text availability were excluded. This review was registered in PROSPERO (CRD42021194468).

RESULTS 59 studies were included with a total of 698 patients. The most common cardiovascular findings were the presence of cardiogenic shock (37\%) and hypotension (8.5\%). Almost all laboratory values were deranged. Cardiac computed tomography scan mostly showed normal results $(56 \%)$, followed by cardiomegaly with pericardial effusion (14\%). Electrocardiography showed normal findings (46\%), ST-segment abnormalities (32\%), and abnormal T wave (12\%). Echocardiography findings showed left ventricle dysfunction (40.6\%), which can be considered most significant, followed by pericardial effusion together with pericarditis (11.4\%) and tricuspid regurgitation (6.9\%).

CONCLUSIONS This review found various cardiac abnormalities that may develop during PIMS-TS. Due to these findings, we should be more vigilant and not underestimate the consequences in pediatric COVID-19 patients.

KEYWORDS cardiovascular, COVID-19, echocardiography, pediatrics, PIMS-TS
Coronavirus disease 2019 (COVID-19) cases have been increasing globally since first emerged in 2019 . As of July 29, 2021, over 195 million cases of COVID-19 have been confirmed, with more than 4 million deaths, ${ }^{1}$ including children. In Indonesia, the case fatality rate in children had reached 1.4, which is very high in the pediatric population. ${ }^{2}$ Children with COVID-19 have a relatively mild infection compared with adults. ${ }^{3,4}$ They also have lower COVID-19 mortality at 0.17 per 100,000 population as of February 2021.5 Children with COVID-19 may have higher hospitalization and mortality rate up to 10 times greater, called pediatric inflammatory multisystem syndrome temporally associated with COVID-19 (PIMS-TS) ${ }^{6}$ or multiple 
inflammatory syndrome in children (MIS-C). ${ }^{7,8}$ These two terms differ in which the latter requires COVID-19 evidence or at least close contact with COVID-19 patients.

PIMS-TS is a rare syndrome that shares standard features with other pediatric inflammatory conditions ${ }^{9}$; this includes the involvement of other organ system dysfunctions such as gastrointestinal, respiratory, nervous, and cardiovascular systems. ${ }^{10-12}$ The dysfunction of these organs could still linger, even though PIMS-TS has been treated. The cardiovascular system is one of the most critically affected organ systems, which can cause long-term symptoms such as chronic fatigue, dyspnea, and chest pain. It would indeed affect the quality of life of children that were affected by PIMS-TS. ${ }^{13,14}$

Several studies have reported PIMS-TS cases with their cardiac manifestations, but the results were varied. ${ }^{15,16}$ Acute cardiac decompensation due to hyperinflammation in patients with MIS-C results in longer hospital stay and higher mortality. ${ }^{17,18}$ Hence, it is crucial to detect cardiovascular abnormalities to improve patients' outcomes. To the best of our knowledge, only few studies have synthesized the cardiology symptoms, laboratory findings, and echocardiography characteristics in children with PIMS-TS. Furthermore, the longterm cardiac sequelae in PIMS-TS patients are still unknown. Thus, this review aimed to summarize the overall symptoms, laboratory, and diagnostic workup findings in PIMS-TS patients, focusing on the cardiovascular manifestations.

\section{METHODS}

This systematic review followed the Preferred Reporting Items for Systematic Review and MetaAnalyses 2020 statement. ${ }^{19}$ The protocol has been registered into the International Prospective Register of Systematic Reviews (PROSPERO) database (CRD42021194468).

The literature search was limited to studies published from December 2020 to October 2021, without language restrictions. All case reports, case series, cross-sectional studies, cohort studies, and possible clinical trials that studied the effects of PIMSTS in pediatric cardiology patients with COVID-19 (aged $0-18$ years) were included in this review. Exclusion criteria comprised MIS-C, animal studies, and studies without full-text references. Abstracts, letters to the editor, and reviews were screened for references to ensure literature saturation before they were excluded.

The literature search was done on November 8 , 2021. The authors utilized four distinct databases, including PubMed, Science Direct, Medline, and Scielo and four different preprint databases, including Medrxiv, Research Square, SSRN, and Biorxiv. Differences in databases may be due to different types of studies, population variations, and case severity. PubMed indexed "ahead of print" articles. Therefore, the latest articles sometimes appear in PubMed but not in Medline. The keywords included "pediatric" AND ("PIMS-TS" $O R$ "pediatric inflammatory multisystem syndrome temporally associated with SARS-CoV-2") AND ("cardiac" OR "cardiology" OR "echocardiography" OR "myocarditis" OR "heart failure"). Data were compiled in a standardized format, including study citations, demographic characteristics of the included participants (age, sex, and comorbidities), severe acute respiratory syndrome coronavirus 2 test results, signs and symptoms, laboratory results, treatments, length of stay, and outcomes. Cardiacspecific examinations such as computed tomography (CT) scan, echocardiography, and electrocardiogram (ECG) were also obtained from each study. If some data were missing, an email would be sent to the corresponding author.

Four independent reviewers (GSO, FM, $\mathrm{RSH}$, and (LB) conducted the initial search and quality assessment of each study. The Joanna Briggs Institute's (JBI) ${ }^{20}$ essential evaluation checklist for case reports was used to measure the general consistency of case series and case reports. Meanwhile, the Newcastle-Ottawa quality assessment scale (NOS)21 was assessed for crosssectional and longitudinal studies. Any discrepancies between JBI and NOS assessments were discussed until a conclusion was reached. Any unresolved disagreements would be consulted with two expert reviewers (RM and $A J)$. The included case reports should fulfill most of the JBI criteria and score $\geq 7$ in the NOS score.

Pooled descriptive tests were used to combine all data in this review. Data presented in median and range (or interquartile range) were converted into mean and standard deviation (SD). All the means and 
SDs were then combined into a single value using the Cochrane method. ${ }^{22}$

\section{RESULTS}

There are 59 studies included in this review, with the selection process is shown in Figure 1. All individual studies achieved good results in JBI and NOS scores, and each study is listed in Table 1 . A total of 698 patients were included, with a mean (SD) age of 9.2 (4.1) years and male predominance (58.0\%). The demographic characteristics of the patients are shown in Table 2. Most patients had positive polymerase chain reaction tests.

Nutritional problems such as underweight or obesity were the most common comorbidities, followed by respiratory problems and neurologic disorders (Table 2). Many patients experienced a shock. The mean (SD) length of hospital stay was 9.8 (11.3) days. Intravenous immunoglobulin was commonly used for PIMS-TS. Of 600 patients with available data on mortality, 3.33\% died.
The mean (SD) of systolic and diastolic blood pressure were 81 (14) and 46 (12), respectively. Almost all laboratory values were deranged in PIMS-TS patients (Table 2). Notably, there was an increase in white blood cells count, neutrophil, C-reactive protein (CRP), ferritin, procalcitonin, creatinine kinase, creatinine, alanine transaminase, aspartate aminotransferase, D-dimer, fibrinogen, and erythrocyte sedimentation rate, and almost all cardiac markers were also elevated. Meanwhile, hemoglobin and lymphocyte values were decreased (Table 2).

The reference range in this table followed the normal values for 9-years-old children (mean age of this study) that were obtained from the Nelson Textbook of Pediatrics ${ }^{23}$ and Mosby's Manual of Diagnostic and Laboratory Tests. ${ }^{24}$ High sensitivity troponin $T$ values were taken from Calò Carducci et al..$^{25}$

The most common cardiac CT scan result was normal (56\%), followed by cardiomegaly with pericardial effusion (14\%). Among 121 patients, the ECG evaluation results were mostly normal (46\%). ST abnormalities (32\%) and abnormal T wave
Identification of studies via databases and registers

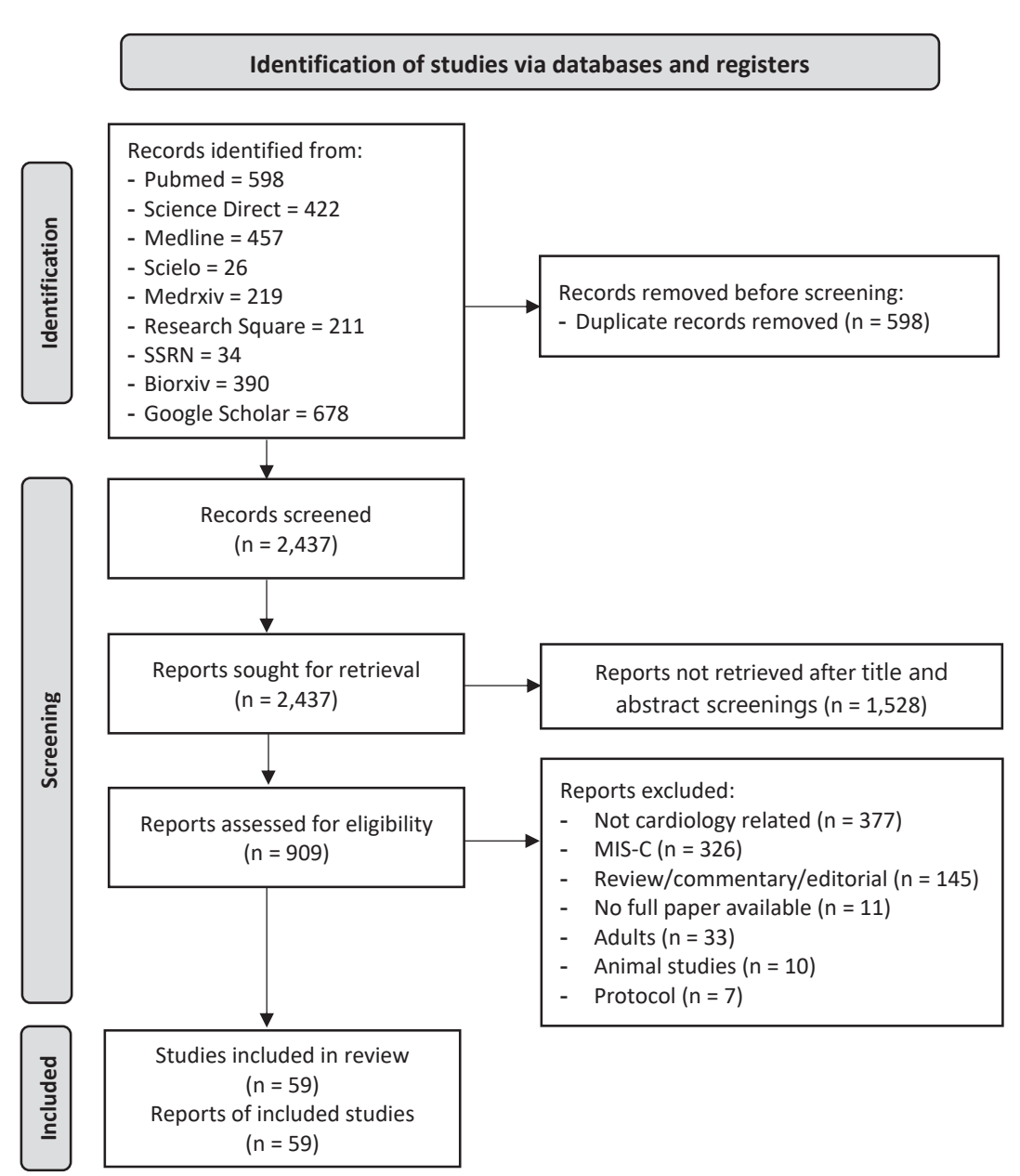

Figure 1. Flow diagram of studies selection

mji.ui.ac.id 


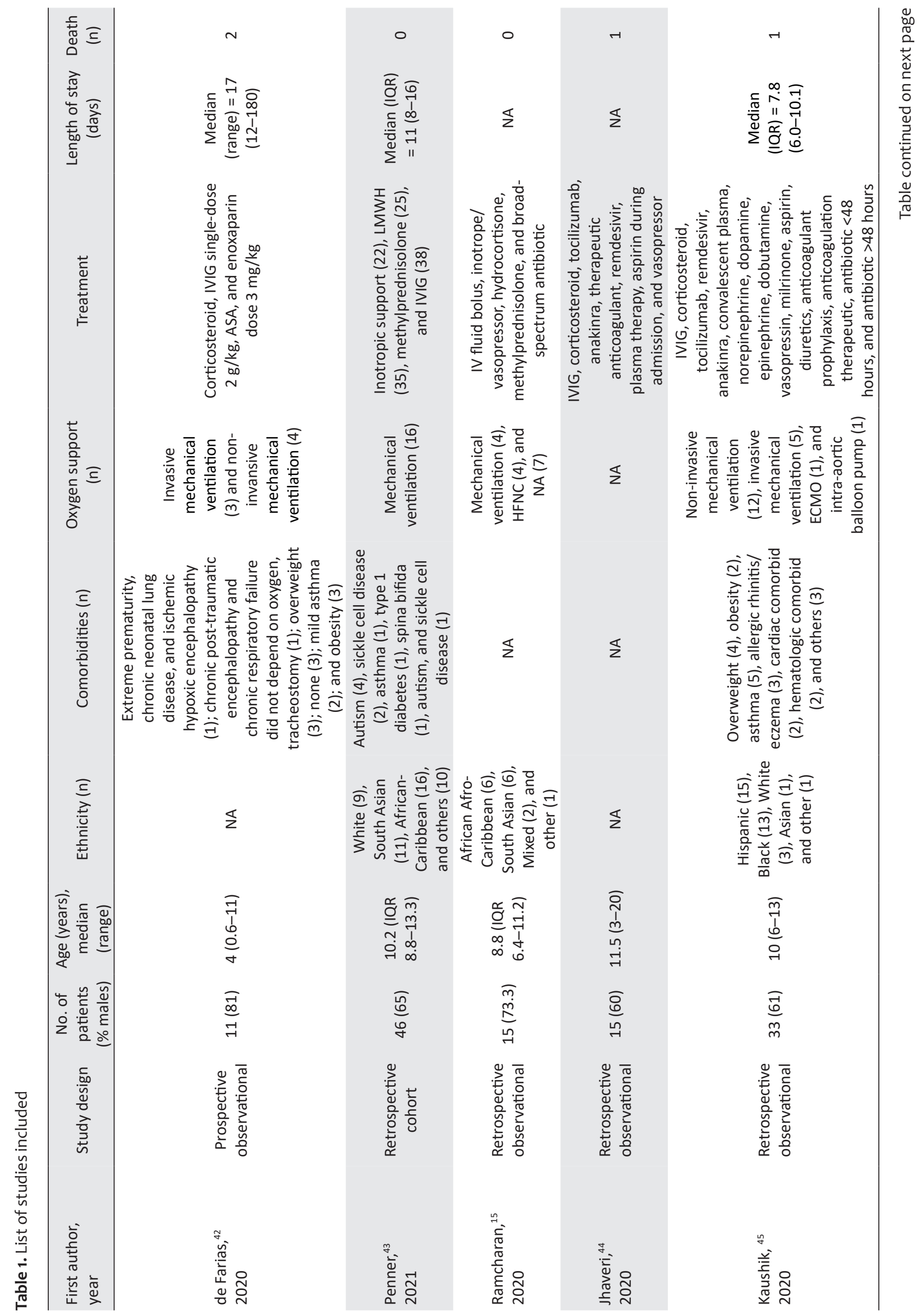




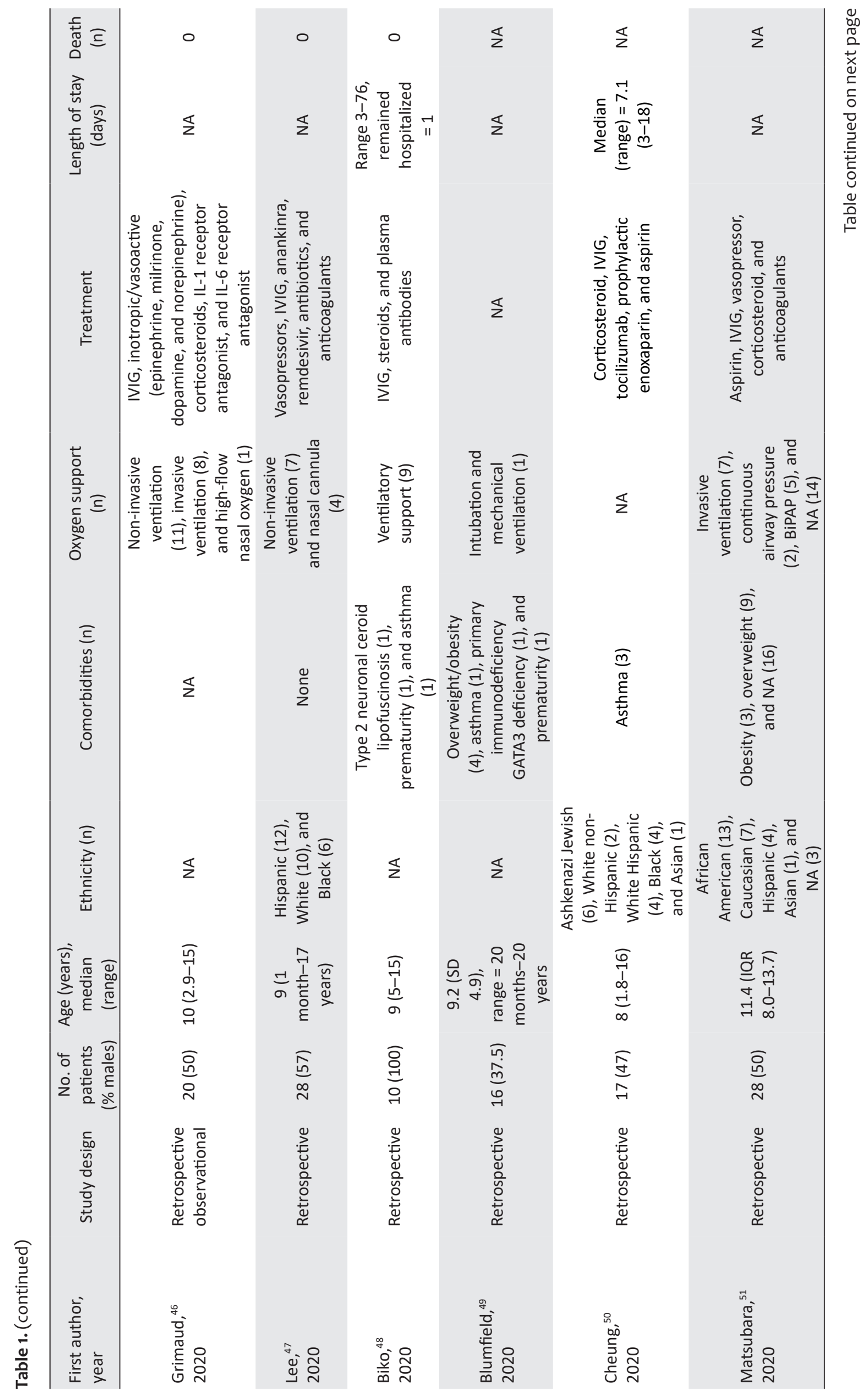




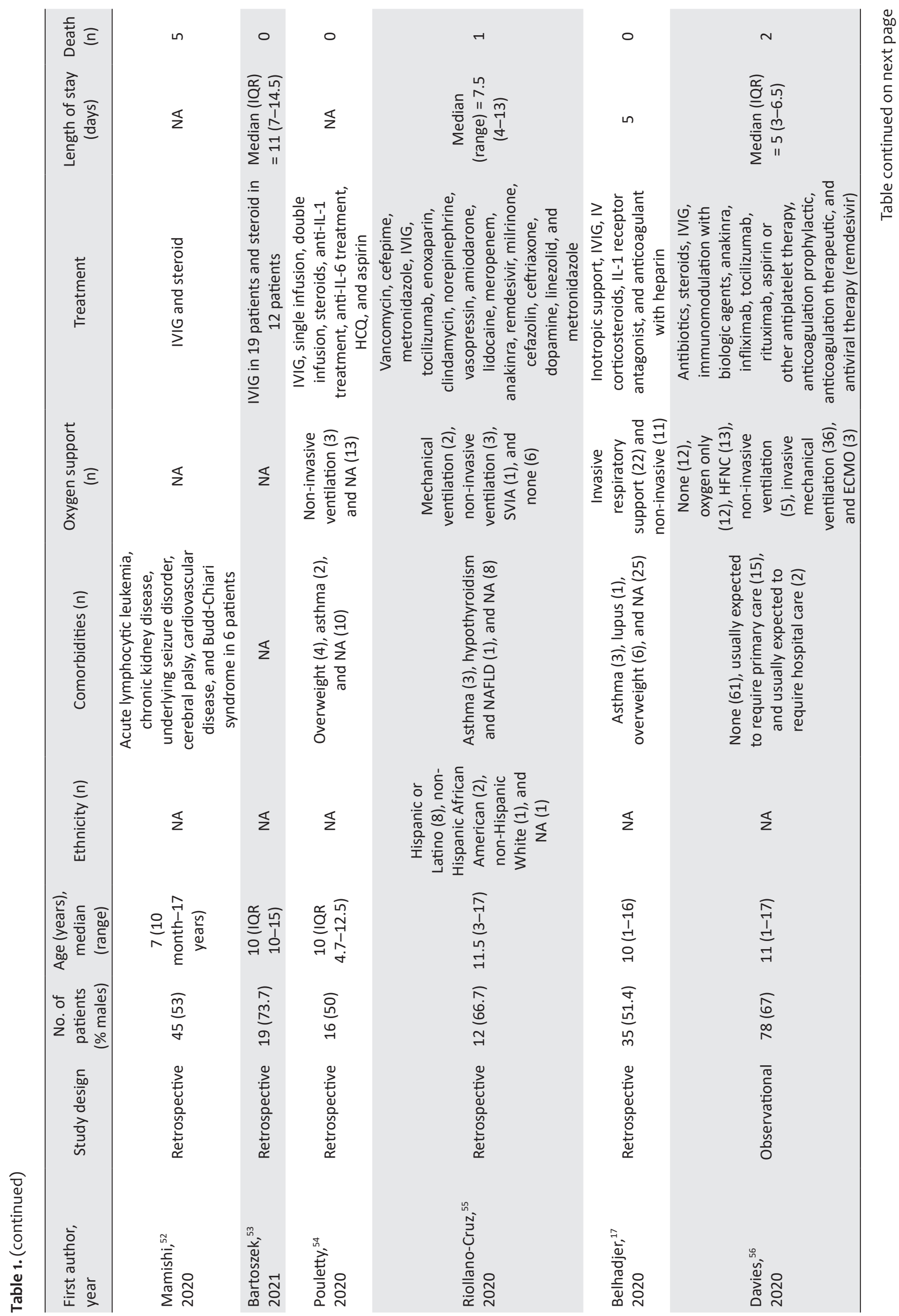




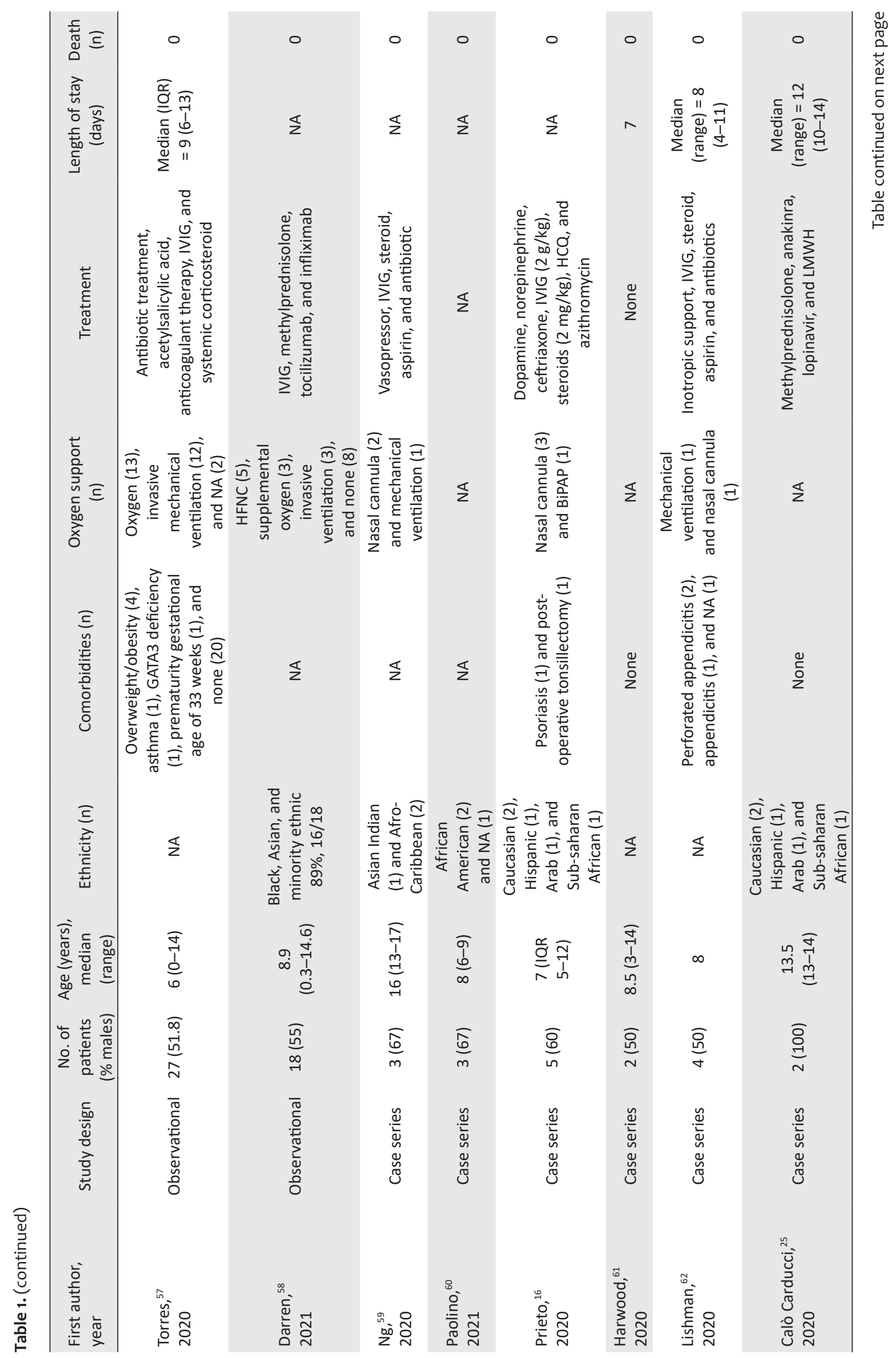




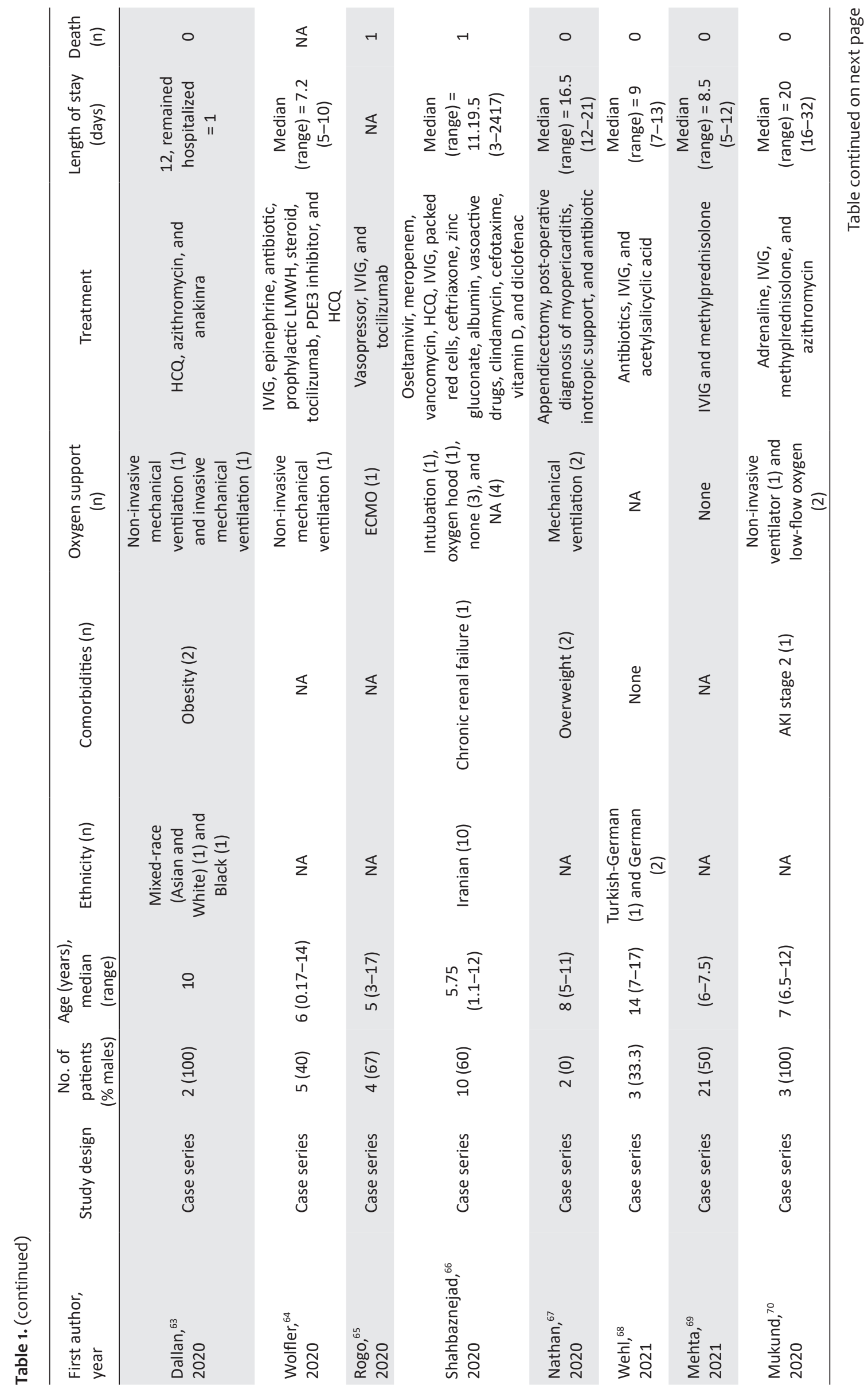




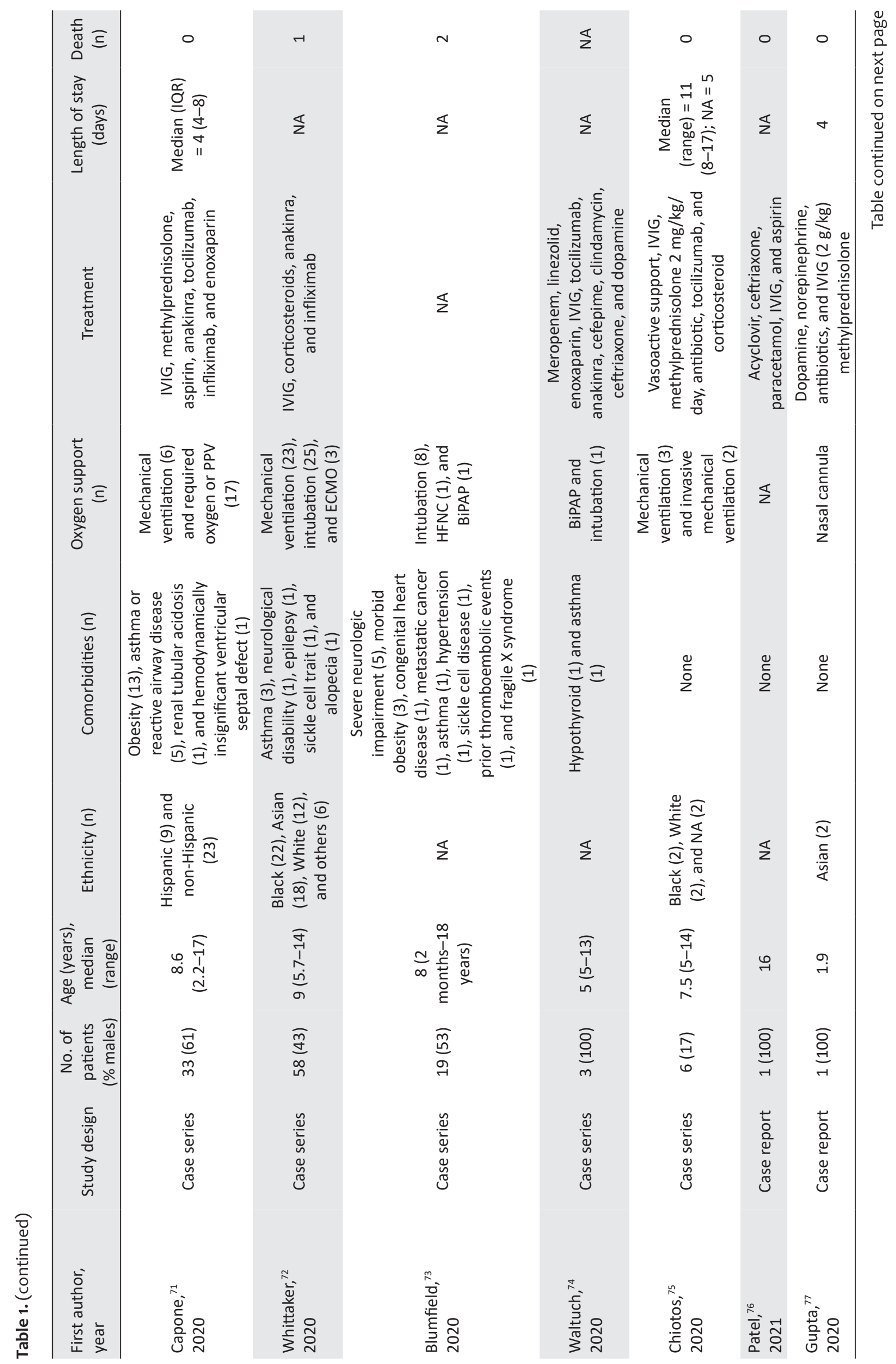




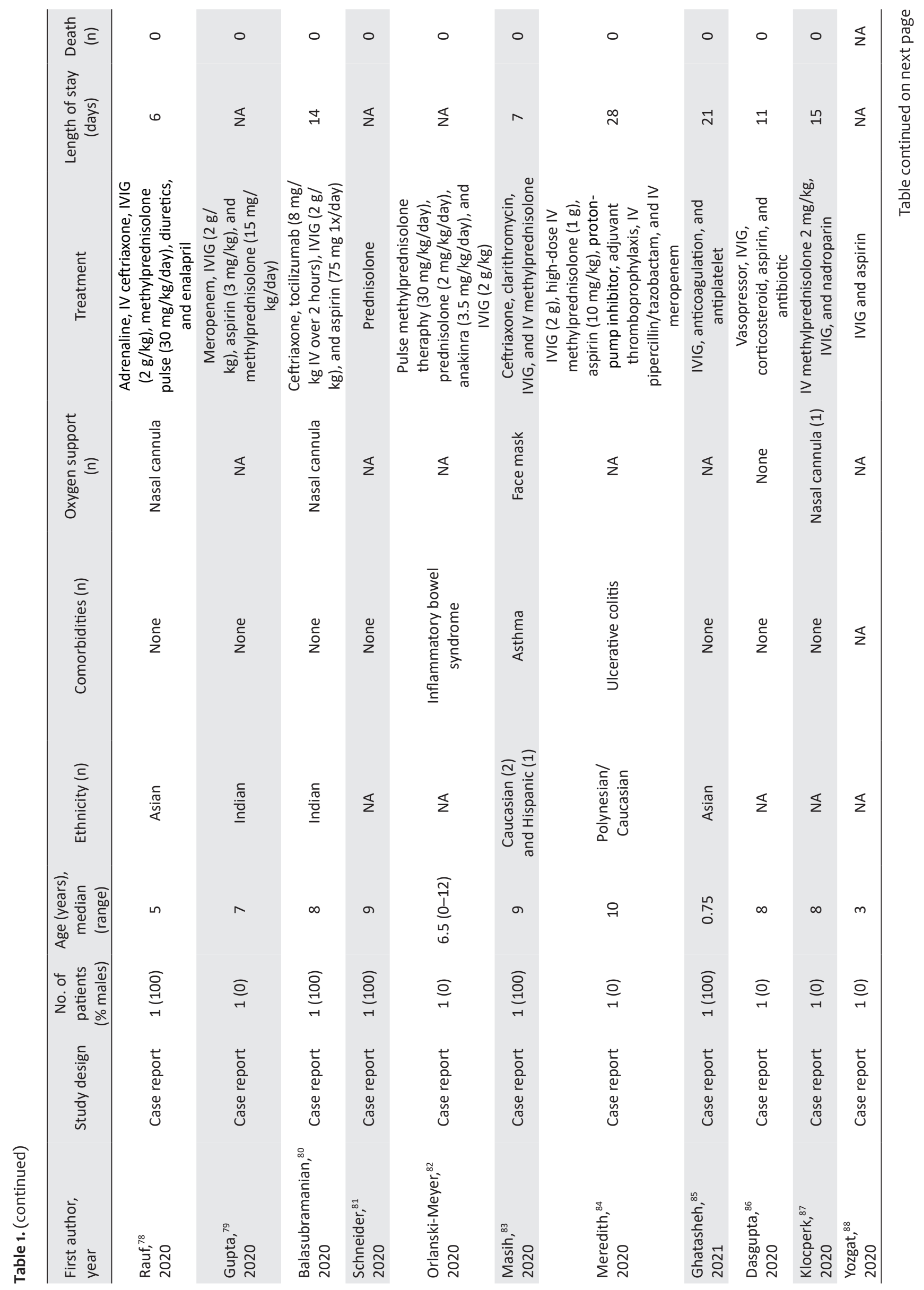




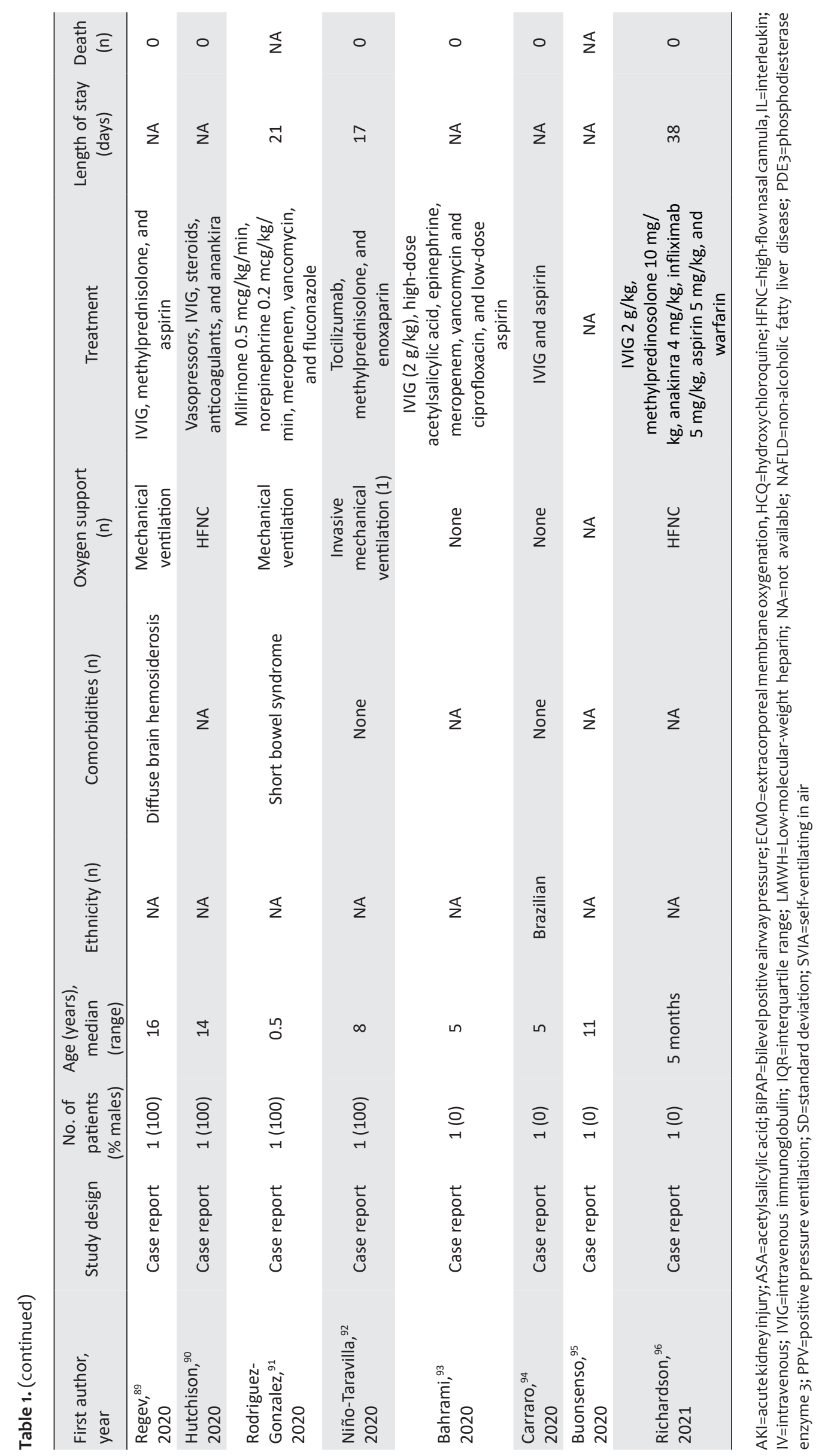


Table 2. Demographic and clinical characteristics, supporting examination, and treatment in PIMS-TS patients

\begin{tabular}{|c|c|c|}
\hline Variables & $\mathrm{n}(\%)$ & $\begin{array}{l}\text { Reference } \\
\text { range }\end{array}$ \\
\hline Age (years), mean $(S D)(n=698)$ & $9.2(4.1)$ & - \\
\hline Male gender $(n=698)$ & $405(58.0)$ & - \\
\hline Ethnicity $(n=404)$ & & - \\
\hline $\begin{array}{l}\text { African American/ } \\
\text { Afro-Caribbean/African }\end{array}$ & $135(33.4)$ & \\
\hline White/European/Caucasian & $71(17.6)$ & \\
\hline Hispanic/Latino & $53(13.1)$ & \\
\hline Asian/Indian/Middle Eastern & $81(20.0)$ & \\
\hline Others & $64(15.8)$ & \\
\hline $\begin{array}{l}\text { Days of fever before admission, } \\
\text { mean (SD) }(n=146)\end{array}$ & $6.27(2.7)$ & - \\
\hline $\begin{array}{l}\text { Temperature upon admission } \\
\left({ }^{\circ} \mathrm{C}\right) \text {, mean }(\mathrm{SD})(\mathrm{n}=51)\end{array}$ & $39(2)$ & - \\
\hline $\begin{array}{l}\text { Systolic blood pressure }(\mathrm{mmHg}) \\
\text { mean }(\mathrm{SD})(\mathrm{n}=42)\end{array}$ & $81(14)$ & - \\
\hline $\begin{array}{l}\text { Diastolic blood pressure } \\
(\mathrm{mmHg}) \text {, mean }(S D)(n=36)\end{array}$ & $46(12)$ & - \\
\hline Signs and symptoms* $(n=698)$ & & - \\
\hline Fever & $563(80.6)$ & \\
\hline Dyspnea & $7(1.0)$ & \\
\hline Tachypnea & $30(4.3)$ & \\
\hline Cough & $85(12.2)$ & \\
\hline Oxygen desaturation & $13(1.9)$ & \\
\hline Hypotension & $59(8.5)$ & \\
\hline Shock & $251(36.0)$ & \\
\hline SARS-CoV-2 test $*(n=654)$ & & - \\
\hline IgM positive & $2(0.3)$ & \\
\hline IgG positive & $181(27.7)$ & \\
\hline IgA positive & $2(0.3)$ & \\
\hline $\lg M$ and IgG positive & $11(1.6)$ & \\
\hline $\lg$ and IgA positive & $41(6.3)$ & \\
\hline $\lg M$, IgG, and IgA positive & $13(1.9)$ & \\
\hline Negative & $122(18.7)$ & \\
\hline $\begin{array}{l}\text { Polymerase chain reaction } \\
\text { test positive }\end{array}$ & $244(37.3)$ & \\
\hline Not specified $^{+}$ & $185(28.3)$ & \\
\hline Comorbidities* $(n=319)$ & & - \\
\hline Respiratory & $34(10.7)$ & \\
\hline Neurologic & $11(3.4)$ & \\
\hline Endocrine & $2(0.6)$ & \\
\hline Gastrointestinal & $9(2.8)$ & \\
\hline Cardiovascular & $6(1.8)$ & \\
\hline Nephrology & $4(1.2)$ & \\
\hline Hemato-oncology & $7(2.1)$ & \\
\hline
\end{tabular}

Table 2. (continued)

\begin{tabular}{lcc}
\hline Variables & $\mathrm{n}(\%)$ & $\begin{array}{c}\text { Reference } \\
\text { range }\end{array}$ \\
\hline Nutritional issues & $62(19.4)$ & \\
Immunology/allergic & $8(2.5)$ \\
Prematurity & $3(0.9)$ \\
Others & $23(7.2)$
\end{tabular}

\section{Length of stay (days), mean (SD)}

$$
(\mathrm{n}=328)
$$

$$
\text { Outcome (alive) ( } n=529)
$$$$
509 \text { (96.2) }
$$

Treatments received* $(n=621)$

Intravenous
immunoglobulin

Vasopressor

469 (75.5)

Antibiotics

Anticoagulants

Diuretic $206(33.2)$

Nasal cannula for oxygen delivery

\begin{tabular}{|c|c|c|}
\hline Non-invasive ventilation & $88(14.2)$ & \\
\hline Mechanical ventilation & $167(26.9)$ & \\
\hline Antiarrhythmics & $1(0.2)$ & \\
\hline Remdesivir & $17(2.7)$ & \\
\hline Hydroxychloroquine & $11(1.8)$ & \\
\hline Corticosteroids & $303(48.8)$ & \\
\hline Aspirin & $166(26.7)$ & \\
\hline Convalescent plasma & $11(1.8)$ & \\
\hline Anakinra & $47(7.6)$ & \\
\hline Tocilizumab & $47(7.6)$ & \\
\hline Laboratory findings & & - \\
\hline \multicolumn{3}{|l|}{ Hematology } \\
\hline $\begin{array}{l}\text { Hemoglobin }(g / d l)(n=204) \text {, } \\
\text { mean (SD) }\end{array}$ & $9.6(2.4)$ & $11.5-14.5$ \\
\hline $\begin{array}{l}\text { White blood cell count } \\
\left(10^{3} / \mu \mathrm{l}\right)(\mathrm{n}=187) \text {, mean }(\mathrm{SD})\end{array}$ & $13.9(6.2)$ & $4-12$ \\
\hline $\begin{array}{l}\text { Lymphocyte }(\%)(n=87) \text {, } \\
\text { mean (SD) }\end{array}$ & $13(13)$ & $25-33$ \\
\hline $\begin{array}{l}\text { Neutrophil }(\%)(n=67) \text {, } \\
\text { mean (SD) }\end{array}$ & $74(19)$ & $57-67$ \\
\hline $\begin{array}{l}\text { Platelets }\left(10^{3} / \mu \mathrm{l}\right)(\mathrm{n}=250) \text {, } \\
\text { mean }(\mathrm{SD})\end{array}$ & $165.7(94.3)$ & $150-400$ \\
\hline
\end{tabular}

Inflammatory markers

\begin{tabular}{lcc} 
& & Male $=$ \\
C-reactive protein $(\mathrm{mg} / \mathrm{l})$ & 218.3 & $0.6-7.9$ \\
$(\mathrm{n}=310)$, mean $(\mathrm{SD})$ & $(119.6)$ & $\begin{array}{c}\text { Female }= \\
\end{array}$ \\
& & $0.5-10.0$ \\
$\begin{array}{l}\text { Ferritin }(\mu \mathrm{g} / \mathrm{ml})(\mathrm{n}=244), \\
\text { mean }(\mathrm{SD})\end{array}$ & 918.5 & $10-60$ \\
\hline
\end{tabular}


Table 2. (continued)

\begin{tabular}{|c|c|c|}
\hline Variables & $\mathrm{n}(\%)$ & $\begin{array}{l}\text { Reference } \\
\text { range }\end{array}$ \\
\hline $\begin{array}{l}\text { Procalcitonin }(\mu \mathrm{g} / \mathrm{ml}) \\
(\mathrm{n}=80) \text {, mean }(\mathrm{SD})\end{array}$ & $42(78)$ & $\leq 0.15$ \\
\hline $\begin{array}{l}\text { Lactate dehydrogenase }(U / \mathrm{I}) \\
(\mathrm{n}=120) \text {, mean }(S D)\end{array}$ & $\begin{array}{c}663.1 \\
(280.9)\end{array}$ & $150-500$ \\
\hline $\begin{array}{l}\text { Creatine kinase }(U / I) \\
(n=42) \text {, mean }(S D)\end{array}$ & $145(221)$ & $5-130$ \\
\hline \multicolumn{3}{|l|}{ Liver and kidney functions } \\
\hline $\begin{array}{l}\text { Creatinine }(m g / d l)(n=233) \text {, } \\
\text { mean }(S D)\end{array}$ & $\begin{array}{c}13.15 \\
(25.25)\end{array}$ & $0.3-0.7$ \\
\hline $\begin{array}{l}\text { Alanine transaminase }(\mathrm{U} / \mathrm{I}) \text {, } \\
\text { mean }(\mathrm{SD})\end{array}$ & $103(217)$ & $5-45$ \\
\hline $\begin{array}{l}\text { Aspartate aminotransferase } \\
(\mathrm{U} / \mathrm{I})(\mathrm{n}=83) \text {, mean }(\mathrm{SD})\end{array}$ & $95(161)$ & $15-50$ \\
\hline $\begin{array}{l}\text { Blood urea nitrogen }(\mathrm{mg} / \mathrm{dl}) \\
(\mathrm{n}=9) \text {, mean }(\mathrm{SD})\end{array}$ & $55(35)$ & $5-18$ \\
\hline \multicolumn{3}{|l|}{ Coagulation } \\
\hline $\begin{array}{l}\text { D-dimer }(\mathrm{mg} / \mathrm{l})(\mathrm{n}=158) \text {, } \\
\text { mean }(\mathrm{SD})\end{array}$ & $\begin{array}{c}2,923.59 \\
(3,170)\end{array}$ & $<0.4$ \\
\hline $\begin{array}{l}\text { Fibrinogen }(\mathrm{mg} / \mathrm{dl})(\mathrm{n}=214) \text {, } \\
\text { mean }(\mathrm{SD})\end{array}$ & $\begin{array}{c}434.26 \\
(293.77)\end{array}$ & $220-440$ \\
\hline $\begin{array}{l}\text { Erythrocyte sedimentation } \\
\text { rate }(\mathrm{mm} / \mathrm{h})(\mathrm{n}=71) \\
\text { mean }(\mathrm{SD})\end{array}$ & $69(29.0)$ & $0-20$ \\
\hline \multicolumn{3}{|l|}{ Cardiac } \\
\hline $\begin{array}{l}\text { Troponin I }(n g / I)(n=27) \text {, } \\
\text { mean }(S D)\end{array}$ & $\begin{array}{c}1,442 \\
(5,693)\end{array}$ & $<300$ \\
\hline $\begin{array}{l}\text { Troponin T (ng/l) }(n=94) \text {, } \\
\text { mean (SD) }\end{array}$ & $98(182)$ & $<100$ \\
\hline $\begin{array}{l}\text { High sensitivity troponin I } \\
(n g / l)(n=1) \text {, value }\end{array}$ & 29 & $<100$ \\
\hline $\begin{array}{l}\text { High sensitivity troponin } T \\
(\mathrm{ng} / \mathrm{l})(\mathrm{n}=2) \text {, mean }(\mathrm{SD})\end{array}$ & $\begin{array}{l}129.75 \\
(43.25)\end{array}$ & $<14$ \\
\hline $\begin{array}{l}\text { Unspecified troponin ( } n g / l) \\
(n=60) \text {, mean (SD) }\end{array}$ & $\begin{array}{c}1,425 \\
(4,260)\end{array}$ & $<10$ \\
\hline $\begin{array}{l}\mathrm{BNP}(\mathrm{pg} / \mathrm{ml})(\mathrm{n}=41) \\
\text { mean }(\mathrm{SD})\end{array}$ & $\begin{array}{c}3,224 \\
(5,038)\end{array}$ & $0-100$ \\
\hline $\begin{array}{l}\text { NT-proBNP }(p g / m l)(n=13) \text {, } \\
\text { mean (SD) }\end{array}$ & $\begin{array}{c}12,323 \\
(12,150)\end{array}$ & $0-450$ \\
\hline $\begin{array}{l}\text { Echocardiography findings } \\
(n=490)\end{array}$ & & - \\
\hline Mitral regurgitation & $31(6.3)$ & \\
\hline Tricuspid regurgitation & $34(6.9)$ & \\
\hline $\begin{array}{l}\text { Pericardial effusion + } \\
\text { pericarditis }\end{array}$ & $56(11.4)$ & \\
\hline Myocarditis & $29(5.9)$ & \\
\hline RV dysfunction & $5(1.0)$ & \\
\hline $\begin{array}{l}\text { Biventricular systolic } \\
\text { dysfunction }\end{array}$ & $3(0.6)$ & \\
\hline Coronary echogenicity & $18(3.7)$ & \\
\hline
\end{tabular}

Table 2. (continued)

\begin{tabular}{|c|c|c|}
\hline Variables & $\mathrm{n}(\%)$ & $\begin{array}{l}\text { Reference } \\
\text { range }\end{array}$ \\
\hline Coronary artery dilatation & $27(5.5)$ & \\
\hline Coronary artery aneurysm & $26(5.3)$ & \\
\hline Aortic regurgitation & $4(0.8)$ & \\
\hline Pulmonary regurgitation & $2(0.4)$ & \\
\hline $\begin{array}{l}\text { Coronary artery dilatation or } \\
\text { aneurysm }\end{array}$ & $18(3.7)$ & \\
\hline Ectatic coronary artery & $15(3.1)$ & \\
\hline Myocardial dysfunction & $5(1.0)$ & \\
\hline Cardiomegaly & $10(2.0)$ & \\
\hline Dilated left ventricle & $3(0.6)$ & \\
\hline Diastolic dysfunction & $5(1.0)$ & \\
\hline Left ventricle dysfunction & $199(40.6)$ & \\
\hline $\operatorname{LVEF}(\mathrm{n}=281)$ & & - \\
\hline$\geq 55 \%$ & $82(29.2)$ & \\
\hline$<55 \%$ & $199(70.8)$ & \\
\hline LCA Z-score $(n=12)$ & & - \\
\hline$<2$ & $8(67)$ & \\
\hline $2-2.5$ & $0(0)$ & \\
\hline$\geq 2.5$ & $4(33)$ & \\
\hline LAD Z-score $(n=6)$ & & - \\
\hline$<2$ & $4(67)$ & \\
\hline $2-2.5$ & $0(0)$ & \\
\hline$\geq 2.5$ & $2(33)$ & \\
\hline RCA Z-score $(n=18)$ & & - \\
\hline$<2$ & $8(44)$ & \\
\hline $2-2.5$ & $1(6)$ & \\
\hline$\geq 2.5$ & $9(50)$ & \\
\hline
\end{tabular}

$B N P=$ brain natriuretic peptide; Ig=immunoglobulin; $L A D=l$ eft anterior descending artery; LCA=left coronary artery; LVEF=left ventricular ejection fraction; NT-proBNP=N-terminal-pro hormone BNP; PIMS-TS=pediatric inflammatory multisystem syndrome temporally associated with COVID-19; RCA=right coronary artery; $\mathrm{RV}=$ right ventricle; SARS-CoV-2=severe acute respiratory syndrome coronavirus 2; $S D=$ standard deviation. *Each patient may have more than one test done/symptoms/comorbidities/treatment; ${ }^{\dagger}$ presented as serologies positive without further specifications

(12\%) were the most common abnormalities found in ECG (results not shown). Table 2 shows the echocardiographic findings on PIMS-TS patients, with left ventricle dysfunction (40.6\%) being the most common abnormality, followed by pericardial effusion together with pericarditis (11.4\%) and tricuspid regurgitation (6.9\%). Echocardiographic findings showed that abnormalities were resolved in 88 days and ectatic coronary arteries in only 3 days 
(results are not shown in the table). Most patients presented with left ventricular ejection fraction (LVEF) of $<55 \%$ (70.8\%), left coronary Z-score of $<2$ $(67 \%)$, left anterior descending artery Z-score of $<2$ $(67 \%)$, and right coronary artery Z-score of $\geq 2.5$ (50\%).

\section{DISCUSSION}

We found that PIMS-TS is most prevalent among older children, and other studies have found a similar mean age of 7 to 10 years. ${ }^{15,26-28}$ Although they share similar clinical features, Kawasaki disease (KD) primarily affects children under 5 years old, with a median age of 2 years. ${ }^{27,29-31}$ Compared with KD, patients with PIMSTS are more likely to present with gastrointestinal symptoms, such as abdominal symptoms, diarrhea, vomiting, and multiorgan involvement. ${ }^{28}$

Shock and hypotension are the two most common signs of cardiovascular system as ventricular dysfunction is frequently encountered in PIMS-TS patients. $^{32}$ Previous studies found cardiovascular symptoms in $71 \%$ of patients. ${ }^{27}$ Most myocardial involvement is usually moderate to severe, which is higher than in KD. ${ }^{28}$

This review also reported inflammatory markers, particularly CRP as the most notable abnormalities in laboratory measurements. This reflects the hyperinflammatory nature of PIMS-TS, 33 as shown in other studies. ${ }^{27,34-37}$ D-dimer and cardiac markers, such as troponin and brain natriuretic peptide, were also found to be elevated across all studies, confirming that myocardial involvement is indeed a hallmark feature of this disease. $34,37,38$

Since PIMS-TS frequently involves the heart, ${ }^{39}$ it is imperative to evaluate the patient's cardiac anatomy and function using echocardiography, cardiac CT, magnetic resonance imaging, and electrocardiography. In this systematic review, most patients had some cardiac involvements with a wide range of echocardiographic manifestations of PIMS-TS. The most common echocardiography abnormalities were reduced left ventricular (LV) function, pericardial effusion with pericarditis, myocarditis, and valvular abnormalities, which are similar to other systematic reviews. ${ }^{36,38,39}$ Patients with impaired left ventricle function, specifically impaired LV global longitudinal strain and LV apical four-chamber peak longitudinal strain at clinical presentation, are at higher risk for developing adverse acute clinical course. Subclinical left myocardial dysfunction may persist for weeks after recovery in these patients. Thus, LV strain may be used to identify the higher-risk patients. ${ }^{40}$ Coronary artery abnormalities were also found in a significant number of patients in this review, with coronary artery dilatation and aneurysms as the most common abnormalities, which also supported by other reviews. ${ }^{38}$ Interestingly, most CT scans showed no abnormalities, with only a minority of patients manifesting cardiomegaly and pericardial effusion. Therefore, CT scans must be reconsidered to detect cardiovascular manifestation in children because it increases radiation exposure risk without generating significant findings. ST-segment and $\mathrm{T}$ wave abnormalities (32\% and $12 \%$, respectively) were most commonly reported in ECG, although most patients displayed normal ECG.

Cardiac abnormalities due to PIMS-TS, as shown in this review, represent a significant medical challenge that warrants more attention. This cardiac involvement may become a long-term health issue, as shown in a previous study that only $28.3 \%$ of patients had improved LVEF after hospital discharge. ${ }^{39}$ Reduced LVEF may manifest as left-sided heart failure, which may cause fatigue, edema, and fluid retention, leading to a significant impairment on quality of life. ${ }^{41}$ Treatments should aim to minimize the long-term impact of PIMSTS.

There are limitations to this systematic review. Since COVID-19 is still considered an emerging new disease, and the term PIMS-TS is relatively new, the knowledge of COVID-19 and PIMS-TS is constantly evolving and changing rapidly. To date, PIMS-TS has only been described from mid-2020. This review also has minimal cardiovascular clinical findings due to limited data. However, more than half of the patients had one or more cardiac abnormalities on echocardiography, emphasizing that most PIMS-TS patients survived the critical phase, although the longterm complications were not observed. Thus, further research is needed as the delayed complications should not be underestimated. In addition, we could not analyze the clinical and echocardiographic progression of the patients. Many cardiac abnormalities in PIMS-TS patients were lacking proper evaluation and followup. Many patients with PIMS-TS were not evaluated with echocardiography or only evaluated once at admission, which leads to difficulty in evaluating the progress of cardiac abnormalities that have developed. Due to the wide variety of data, we suggest future 
studies to standardize the echocardiographic finding reports associated with PIMS-TS and assess the disease progression.

In conclusion, numerous organ or system dysfunctions may complicate the clinical course of pediatric patients with COVID-19. This review primarily focused on the possible development of various cardiac abnormalities, which were assessed by laboratory tests and imaging, with echocardiography as the leading modality in detecting and evaluating these patients. We should be more vigilant with cardiac abnormalities in COVID-19 pediatric patients and should not underestimate their consequences in pediatric patients. Thus, the importance of addressing cardiac abnormalities that may occur in PIMS-TS can not be understated.

\section{Conflict of Interest}

The authors affirm no conflict of interest in this study.

Acknowledgment

None.

Funding Sources

None.

\section{REFERENCES}

1. World Health Organization. WHO coronavirus (COVID-19) dashboard [Internet]. World Health Organization; 2021 [cited 2021 Jul 29]. Available from: https://covid19.who.int/.

2. Pudjiadi AH, Putri ND, Sjakti HA, Yanuarso PB, Gunardi H, Roeslani RD, et al. Pediatric COVID-19: report from Indonesian Pediatric Society Data Registry. Front Pediatr. 2021;9:716898.

3. Zimmermann P, Curtis N. Why is COVID-19 less severe in children? A review of the proposed mechanisms underlying the age-related difference in severity of SARS-CoV-2 infections. Arch Dis Child. 2021;106:429-39.

4. Zimmermann $P$, Curtis N. Coronavirus infections in children including COVID-19: an overview of the epidemiology, clinical features, diagnosis, treatment and prevention options in children. Pediatr Infect Dis J. 2020;39(5):355-68.

5. Bhopal SS, Bagaria J, Olabi B, Bhopal R. Children and young people remain at low risk of COVID-19 mortality. Lancet Child Adolesc Health. 2021;5(5):e12-3.

6. Fernández-Sarmiento J, De Souza D, Jabornisky R, Gonzalez GA, Arias López MDP, Palacio G. Paediatric inflammatory multisystem syndrome temporally associated with COVID-19 (PIMS-TS): a narrative review and the viewpoint of the Latin American Society of Pediatric Intensive Care (SLACIP) Sepsis Committee. BMJ Paediatr Open. 2021;5(1):e000894.

7. Centers for Disease Control and Prevention. Information for healthcare providers about multisystem inflammatory syndrome in children (MIS-C) [Internet]. Centers for Disease Control and Prevention; 2021 [cited 2021 Aug 3]. Available from: https://www.cdc.gov/mis/hcp/index.html.

8. World Health Organization. Multisystem inflammatory syndrome in children and adolescents temporally related to COVID-19 [Internet]. World Health Organization; 2020 [cited $2021 \mathrm{Jul}$ 29]. Available from: https://www.who.int/news-room/ commentaries/detail/multisystem-inflammatory-syndrome-in- children-and-adolescents-with-covid-19.

9. Royal Collage of Paediatrics and Child Health. Paediatric multisystem inflammatory syndrome temporally associated with COVID-19 (PIMS) - guidance for clinicians [Internet]. Royal Collage of Paediatrics and Child Health; 2020 [cited 2021 Jul 29]. Available from: https://www.rcpch.ac.uk/resources/paediatricmultisystem-inflammatory-syndrome-temporally-associatedcovid-19-pims-guidance.

10. Godfred-Cato S, Bryant B, Leung J, Oster ME, Conklin L, Abrams J, et al. COVID-19-associated multisystem inflammatory syndrome in children - United States, March-July 2020. MMWR Morb Mortal Wkly Rep. 2020;69(32):1074-80.

11. Feldstein LR, Tenforde MW, Friedman KG, Newhams M, Rose EB, Dapul H, et al. Characteristics and outcomes of US children and adolescents with multisystem inflammatory syndrome in children (MIS-C) compared with severe acute COVID-19. JAMA. 2021;325(11):1074-87.

12. Feldstein LR, Rose EB, Horwitz SM, Collins JP, Newhams MM, Son MBF, et al. Multisystem inflammatory syndrome in U.S. children and adolescents. N Engl J Med. 2020;383:334-46.

13. Carfì A, Bernabei R, Landi F; Gemelli Against COVID-19 PostAcute Care Study Group. Persistent symptoms in patients after acute COVID-19. JAMA. 2020;324(6):603-5.

14. Rodriguez-Gonzalez M, Castellano-Martinez A, CascalesPoyatos HM, Perez-Reviriego AA. Cardiovascular impact of COVID-19 with a focus on children: a systematic review. World J Clin Cases. 2020;8(21):5250-83.

15. Ramcharan T, Nolan O, Lai CY, Prabhu N, Krishnamurthy $R$, Richter $A G$, et al. Paediatric inflammatory multisystem syndrome: temporally associated with SARS-CoV-2 (PIMS-TS): cardiac features, management and short-term outcomes at a UK Tertiary Paediatric Hospital. Pediatr Cardiol. 2020;41(7):1391401.

16. Prieto LM, Toral B, LLorente A, Coca D, Blázquez-Gamero D. Cardiovascular magnetic resonance imaging in children with pediatric inflammatory multisystem syndrome temporally associated with SARS-CoV-2 and heart dysfunction. Clin Microbiol Infect. 2020;27(4):648-50.

17. Belhadjer Z, Méot M, Bajolle F, Khraiche D, Legendre A, Abakka $S$, et al. Acute heart failure in multisystem inflammatory syndrome in children in the context of global SARS-CoV-2 pandemic. Circulation. 2020;142(5):429-36.

18. Acevedo L, Piñeres-Olave BE, Niño-Serna LF, Vega LM, Gomez IJA, Chacón S, et al. Mortality and clinical characteristics of multisystem inflammatory syndrome in children (MIS-C) associated with covid-19 in critically ill patients: an observational multicenter study (MISCO study). BMC Pediatr. 2021;21(1):516.

19. Page MJ, McKenzie JE, Bossuyt PM, Boutron I, Hoffmann TC, Mulrow CD, et al. The PRISMA 2020 statement: an updated guideline for reporting systematic reviews. BMJ. 2021;372:n71.

20. Joanna Briggs Institute. The Joanna Briggs Institute critical appraisal tools for use in JBI systematic reviews: checklists for case reports [Internet]. Joanna Briggs Institute; 2019 [cited $2021 \mathrm{Jul}$ 18]. Available from: https://jbi.global/sites/default/ files/2019-05/JBI_Critical_Appraisal-Checklist_for_Case_ Reports2017_0.pdf.

21. Wells GA, Shea B, O'Connell D, Peterson J, Welch V, Losos M, et al. The Newcastle-Ottawa Scale (NOS) for assessing the quality of nonrandomised studies in meta-analyses [Internet]. The Ottawa Hospital Research Institute; 2014. Available from: http:// www.ohri.ca/programs/clinical_epidemiology/oxford.asp.

22. Wan X, Wang W, Liu J, Tong T. Estimating the sample mean and standard deviation from the sample size, median, range and/or interquartile range. BMC Med Res Methodol. 2014;14:135.

23. Kliegman RM, Geme J St., Blum NJ, Shah SS, Tasker RC, Wilson $\mathrm{KM}$, et al. Nelson textbook of pediatrics. 21st ed. Philadelphia: Elsevier; 2020.

24. Pagana DK, Pagana TJ, Pagana TN. Mosby's diagnostic and laboratory test reference. 12th ed. Botkin C, Bowlder NC, Burd 
EM, Chavalitdhamrong D, Demers LM, Hollensead SC, et al., editors. St. Louis: Elsevier; 2015.

25. Calò Carducci Fl, De Ioris MA, Agrati C, Carsetti R, Perrotta D, D'Argenio $P$, et al. Hyperinflammation in two severe acute respiratory syndrome coronavirus 2-infected adolescents successfully treated with the interleukin-1 inhibitor anakinra and glucocorticoids. Front Pediatr. 2020;8:576912.

26. Dhanalakshmi K, Venkataraman A, Balasubramanian S, Madhusudan M, Amperayani S, Putilibai S, et al. Epidemiological and clinical profile of pediatric inflammatory multisystem syndrome - temporally associated with SARS-CoV-2 (PIMS-TS) in Indian children. Indian Pediatr. 2020;57(11):1010-4.

27. Abrams JY, Godfred-Cato SE, Oster ME, Chow EJ, Koumans EH, Bryant $\mathrm{B}$, et al. Multisystem inflammatory syndrome in children associated with severe acute respiratory syndrome coronavirus 2: a systematic review. J Pediatr. 2020;226:45-54.e1.

28. Shulman ST. Pediatric coronavirus disease-2019-associated multisystem inflammatory syndrome. J Pediatric Infect Dis Soc. 2020;9(3):285-6.

29. Centers for Disease Control and Prevention. Kawasaki disease (KD) [Internet]. Centers for Disease Control and Prevention; 2020 [cited 2021 Aug 20]. Available from: https://www.cdc.gov/ kawasaki/index.html.

30. Newburger J, Takahashi M, Burns J. Kawasaki disease. J Am Coll Cardiol. 2016;67(14):1738-49.

31. Ramphul K, Mejias SG. Kawasaki disease: a comprehensive review. Arch Med Sci Atheroscler Dis. 2018;3:e41-5.

32. Elias MD, McCrindle BW, Larios G, Choueiter NF, Dahdah N, Harahsheh AS, et al. Management of multisystem inflammatory syndrome in children associated with COVID-19: a survey from the international kawasaki disease registry. CJC Open. 2020;2(6):632-40.

33. Consiglio CR, Cotugno N, Sardh F, Pou C, Amodio D, Rodriguez L, et al. The immunology of multisystem inflammatory syndrome in children with COVID-19. Cell. 2020;183(4):968-81.e7.

34. Kaushik A, Gupta S, Sood M, Sharma S, Verma S. A systematic review of multisystem inflammatory syndrome in children associated with SARS-CoV-2 infection. Pediatr Infect Dis J. 2020;39(11):e340-6.

35. Rowley AH, Shulman ST, Arditi M. Immune pathogenesis of COVID-19-related multisystem inflammatory syndrome in children. J Clin Invest. 2020;130(11):5619-21.

36. Ahmed M, Advani S, Moreira A, Zoretic S, Martinez J, Chorath $\mathrm{K}$, et al. Multisystem inflammatory syndrome in children: a systematic review. EClinical Medicine. 2020;26:100527.

37. Radia T, Williams N, Agrawal P, Harman K, Weale J, Cook $J$, et al. Multi-system inflammatory syndrome in children \& adolescents (MIS-C): a systematic review of clinical features and presentation. Paediatr Respir Rev. 2021;38:51-7.

38. Alsaied T, Tremoulet AH, Burns JC, Saidi A, Dionne A, Lang SM, et al. Review of cardiac involvement in multisystem inflammatory syndrome in children. Circulation. 2020;143(1):78-88.

39. Henrina J, Putra ICS, Lawrensia S, Marta DS, Wijaya E, Saboe A, et al. Cardiac manifestations, treatment characteristics, and outcomes of paediatric inflammatory multisystem syndrome temporally associated with severe acute respiratory syndrome coronavirus-2: a systematic review. Prog Pediatr Cardiol. 2021;63:101365.

40. Sanil Y, Misra A, Safa R, Blake JM, Eddine AC, Balakrishnan P, et al. Echocardiographic indicators associated with adverse clinical course and cardiac sequelae in multisystem inflammatory syndrome in children with coronavirus disease 2019. J Am SoC Echocardiogr. 2021;34(8):862-76.

41. Baptist Health. Left-sided heart failure [Internet]. Louisville: Baptist Health; 2021 [cited 2021 Aug 20]. Available from: https:// www.baptisthealth.com/services/heart-care/conditions/leftsided-heart-failure.

42. de Farias ECF, Pedro Piva J, de Mello MLFMF, do Nascimento LMPP, Costa CC, Machado MMM, et al. Multisystem inflammatory syndrome associated with coronavirus disease in children: a multi-centered study in Belém, Pará, Brazil. Pediatr Infect Dis J. 2020;39(11):e374-6.

43. Penner J, Abdel-Mannan O, Grant K, Maillard S, Kucera F, Hassell J, et al. 6-month multidisciplinary follow-up and outcomes of patients with paediatric inflammatory multisystem syndrome (PIMS-TS) at a UK tertiary paediatric hospital: a retrospective cohort study. Lancet Child Adolesc Health. 2021;5(7):473-82.

44. Jhaveri S, Ahluwalia N, Kaushik S, Trachtman R, Kowalsky S, Aydin S, et al. Longitudinal echocardiographic assessment of coronary arteries and left ventricular function following multisystem inflammatory syndrome in children. J Pediatr. 2021;228:290-3.e1.

45. Kaushik S, Aydin SI, Derespina KR, Bansal PB, Kowalsky S, Trachtman R, et al. Multisystem inflammatory syndrome in children associated with severe acute respiratory syndrome coronavirus 2 infection (MIS-C): a multi-institutional study from New York City. J Pediatr. 2020;224:24-9.

46. Grimaud M, Starck J, Levy M, Marais C, Chareyre J, Khraiche D, et al. Acute myocarditis and multisystem inflammatory emerging disease following SARS-CoV-2 infection in critically ill children. Ann Intensive Care. 2020;10:69.

47. Lee PY, Day-Lewis M, Henderson LA, Friedman KG, Lo J, Roberts $\mathrm{JE}$, et al. Distinct clinical and immunological features of SARSCoV-2-induced multisystem inflammatory syndrome in children. J Clin Invest. 2020;130(11):5942-50.

48. Biko DM, Ramirez-Suarez KI, Barrera CA, Banerjee A, Matsubara D, Kaplan SL, et al. Imaging of children with COVID-19: experience from a tertiary children's hospital in the United States. Pediatr Radiol. 2020;51(2):239-47.

49. Blumfield E, Levin TL, Kurian J, Lee EY, Liszewski MC. Imaging findings in multisystem inflammatory syndrome in children (MIS-C) associated with coronavirus disease (COVID-19). AJR Am J Roentgenol. 2021;216(2):507-17.

50. Cheung EW, Zachariah P, Gorelik M, Boneparth A, Kernie SG, Orange JS, et al. Multisystem inflammatory syndrome related to COVID-19 in previously healthy children and adolescents in New York City. JAMA. 2020;324(3):294-6.

51. Matsubara D, Kauffman HL, Wang $\mathrm{Y}$, Calderon-Anyosa R, Nadaraj S, Elias MD, et al. Echocardiographic findings in pediatric multisystem inflammatory syndrome associated with COVID-19 in the United States. J Am Coll Cardiol. 2020;76(17):1947-61.

52. Mamishi S, Movahedi Z, Mohammadi M, Ziaee V, Khodabandeh M, Abdolsalehi MR, et al. Multisystem inflammatory syndrome associated with SARS-CoV-2 infection in 45 children: a first report from Iran. Epidemiol Infect. 2020;148:e196.

53. Bartoszek M, Małek ŁA, Barczuk-Falęcka M, Brzewski M. Cardiac magnetic resonance follow-up of children after pediatric inflammatory multisystem syndrome temporally associated with SARS-CoV-2 with initial cardiac involvement. J Magn Reson Imaging. 2021:10.1002/jmri.2787.

54. Pouletty M, Borocco C, Ouldali N, Caseris M, Basmaci R, Lachaume $\mathrm{N}$, et al. Paediatric multisystem inflammatory syndrome temporally associated with SARS-CoV-2 mimicking Kawasaki disease (Kawa-COVID-19): a multicentre cohort. Ann Rheum Dis. 2020;79(8):999-1006.

55. Riollano-Cruz M, Akkoyun E, Briceno-Brito E, Kowalsky S, Reed J, Posada R, et al. Multisystem inflammatory syndrome in children related to COVID-19: a New York City experience. J Med Virol. 2020;93(1):424-33.

56. Davies P, Evans C, Kanthimathinathan HK, Lillie J, Brierley J, Waters G, et al. Intensive care admissions of children with paediatric inflammatory multisystem syndrome temporally associated with SARS-CoV-2 (PIMS-TS) in the UK: a multicentre observational study. Lancet Child Adolesc Heal. 2020;4(9):66977.

57. Torres JP, Izquierdo G, Acuña M, Pavez D, Reyes F, Fritis A, et al. Multisystem inflammatory syndrome in children (MIS-C): report of the clinical and epidemiological characteristics of cases in 
Santiago de Chile during the SARS-CoV-2 pandemic. Int J Infect Dis. 2020;100:75-81.

58. Darren A, Osman M, Masilamani K, Habib Ali S, Kanthimathinathan HK, Chikermane A, et al. Vitamin D status of children with paediatric inflammatory multisystem syndrome temporally associated with severe acute respiratory syndrome coronavirus 2 (PIMS-TS). Br J Nutr. 2021:1-26.

59. Ng KF, Kothari T, Bandi S, Bird PW, Goyal K, Zoha M, et al. COVID-19 multisystem inflammatory syndrome in three teenagers with confirmed SARS-CoV-2 infection. J Med Virol. 2020;92(11):2880-6.

6o. Paolino J, Williams DA. Peripheral blood smears of children with multisystem inflammatory syndrome demonstrate prominence of early myeloid forms with morphologic evidence of toxic change. Pediatr Blood Cancer. 2021;68(1):e28551.

61. Harwood R, Partridge R, Minford J, Almond S. Paediatric abdominal pain in the time of COVID-19: a new diagnostic dilemma. J Surg Case Reports. 2020;2020(9):rjaa337.

62. Lishman J, Kohler C, de Vos C, van der Zalm MM, Itana J, Redfern $A$, et al. Acute appendicitis in multisystem inflammatory syndrome in children with COVID-19. Pediatr Infect Dis J. 2020;39(12):472-3.

63. Dallan C, Romano F, Siebert J, Politi S, Lacroix L, Sahyoun C. Septic shock presentation in adolescents with COVID-19. Lancet Child Adolesc Health. 2020;4(7):e21-3.

64. Wolfler A, Mannarino S, Giacomet V, Camporesi A, Zuccotti G. Acute myocardial injury: a novel clinical pattern in children with COVID-19. Lancet Child Adolesc Health. 2020;4(8):e26-7.

65. Rogo T, Mathur K, Purswani M. Systemic inflammation with cardiac involvement in pediatric patients with evidence of COVID-19 in a community hospital in the Bronx, New York. J Pediatric Infect Dis Soc. 2020;9(4):502-3.

66. Shahbaznejad L, Navaeifar MR, Abbaskhanian A, Hosseinzadeh F, Rahimzadeh G, Rezai MS. Clinical characteristics of 10 children with a pediatric inflammatory multisystem syndrome associated with COVID-19 in Iran. BMC Pediatr. 2020;20(1):513.

67. Nathan N, Prevost B, Sileo C, Richard N, Berdah L, Thouvenin $\mathrm{G}$, et al. The wide spectrum of COVID-19 clinical presentation in children. J Clin Med. 2020;9(9):2950.

68. Wehl G, Franke J, Frühwirth M, Edlinger M, Rauchenzauner M. Successful treatment of pediatric inflammatory multisystem syndrome temporally associated with COVID-19 (PIMS-TS) with split doses of immunoglobulin $G$ and estimation of PIMS-TS incidence in a county district in southern Germany. Healthcare (Basel). 2021;9(4):481.

69. Mehta R, Ghosh S, Nandy J, Das S, Chattopadhyay A. Atypical presentation of complete heart block in children with pediatric inflammatory multisystem syndrome: a case series of two patients. Ann Pediatr Cardiol. 2021;14(3):408-11.

70. Mukund, Sharma M, Mehta A, Kumar A, Bhat V. Pediatric inflammatory multisystem syndrome temporally associated with severe acute respiratory syndrome coronavirus 2 - an emerging problem of PICU: a case series. J Pediatr Crit Care. 2020;7(5):271-5.

71. Capone CA, Subramony A, Sweberg T, Schneider J, Shah S, Rubin $L$, et al. Characteristics, cardiac involvement, and outcomes of multisystem inflammatory syndrome of childhood associated with severe acute respiratory syndrome coronavirus 2 infection. J Pediatr. 2020;224:141-5.

72. Whittaker E, Bamford A, Kenny J, Kaforou M, Jones CE, Shah $\mathrm{P}$, et al. Clinical characteristics of 58 children with a pediatric inflammatory multisystem syndrome temporally associated with SARS-CoV-2. JAMA. 2020;324(3):259-69.

73. Blumfield E, Levin TL. COVID-19 in pediatric patients: a case series from the Bronx, NY. Pediatr Radiol. 2020;50(10):1369-74.

74. Waltuch T, Gill P, Zinns LE, Whitney R, Tokarski J, Tsung JW, et al. Features of COVID-19 post-infectious cytokine release syndrome in children presenting to the emergency department. Am J Emerg Med. 2020;38(10):2246.e3-6.
75. Chiotos K, Bassiri H, Behrens EM, Blatz AM, Chang J, Diorio C, et al. Multisystem inflammatory syndrome in children during the coronavirus 2019 pandemic: a case series. J Pediatric Infect Dis Soc. 2020;9(3):393-8.

76. Patel ST, Wright H. Lessons of the month: a misunderstood teenager with paediatric inflammatory multisystem syndrome - temporarily associated with SARS-CoV-2 admitted under adult medicine. Clin Med (Lond). 2021;21(1):e96-9.

77. Gupta YK, Shenoy S, Paike K, Tudu MN. Severe Paediatric multisystem inflammatory syndrome (PIMS) in a 23 months old baby post COVID-19 effectively managed with IVIG and pulse steroid: case report. Res Sq. 2020;1-4.

78. Rauf A, Vijayan A, John ST, Krishnan R, Latheef A. Multisystem inflammatory syndrome with features of atypical kawasaki disease during COVID-19 pandemic. Indian J Pediatr. 2020;87(9):745-7.

79. Gupta A, Gill A, Sharma M, Garg M. Multi-system inflammatory syndrome in a child mimicking kawasaki disease. J Trop Pediatr. 2021;67(3):fmaao6o.

80. Balasubramanian S, Nagendran TM, Ramachandran B, Ramanan A V. Hyper-inflammatory syndrome in a child with COVID-19 treated successfully with intravenous immunoglobulin and tocilizumab. Indian Pediatr. 2020;57(7):681-3.

81. Schneider DT, Pütz-Dolderer J, Berrang J. Pediatric multisystemic inflammatory syndrome associated With SARS-CoV-2 infection. Dtsch Arztebl Int. 2020;117(25):431.

82. Orlanski-Meyer E, Yogev D, Auerbach A, Megged O, Glikman $D$, Hashkes PJ, et al. Multisystem inflammatory syndrome in children associated with severe acute respiratory syndrome coronavirus-2 in an 8-week old infant. J Pediatric Infect Dis Soc. 2020;9(6):781-4.

83. Masih M, Moll S, Raza N. Paediatric case of prolonged COVID-19 manifesting as PMIS-TS and atypical Kawasaki. BMJ Case Rep. 2020;13(9):e237194.

84. Meredith J, Khedim CA, Henderson P, Wilson DC, Russell RK. Infliximab attenuates paediatric inflammatory multisystem syndrome in SARS-CoV-2 (PIMS-TS). Preprints 2020, 2020060118

85. Ghatasheh G, Al Dhanhani H, Goyal A, Noureddin MB, Al Awaad $D$, Peerwani Z. COVID-19-related giant coronary aneurysms in an infant with multisystem inflammatory disorder in children: the first case report from the United Arab Emirates and the Arab region. Case Rep Infect Dis. 2021;2021:8872412.

86. Dasgupta K, Finch SE. A case of pediatric multisystem inflammatory syndrome temporally associated with COVID-19 in South Dakota. S D Med. 2020;73(6):246-51.

87. Klocperk A, Parackova Z, Dissou J, Malcova H, Pavlicek P, Vymazal T, et al. Case report: systemic inflammatory response and fast recovery in a pediatric patient with COVID-19. Front Immunol. 2020;11:1665.

88. Yozgat CY, Uzuner S, Bursal Duramaz B, Yozgat Y, Erenberk $U$, Iscan $A$, et al. Dermatological manifestation of pediatrics multisystem inflammatory syndrome associated with COVID-19 in a 3-year-old girl. Dermatol Ther. 2020;33(4):e13770.

89. Regev T, Antemi M, Eytan D, Shachor-Meyouhas Y, Illivitzki A, Aviel $\mathrm{Y}$, et al. Pediatric inflammatory multisystem syndrome with central nervous system involvement and hypocomplementemia following SARS-COV-2 infection. Pediatr Infect Dis J. 2020;39(8):e206-7.

90. Hutchison L, Plichta AM, Lerea Y, Madora M, Ushay HM. Neuropsychiatric symptoms in an adolescent boy with multisystem inflammatory syndrome in children. Psychosomatics. 2020;61(6):739-44.

91. Rodriguez-Gonzalez M, Rodríguez-Campoy $P$, Sánchez-Códez M, Gutiérrez-Rosa I, Castellano-Martinez A, Rodríguez-Benítez A. New onset severe right ventricular failure associated with COVID-19 in a young infant without previous heart disease. Cardiol Young. 2020;30(9):1346-9.

92. Niño-Taravilla C, Espinosa-Vielma YP, Otaola-Arca H, PoliHarlowe C, Tapia LI, Ortiz-Fritz P. Pediatric inflammatory 
multisystem syndrome temporally associated with SARS-CoV-2 treated with tocilizumab. Pediatr Rep. 2020;12(3):142-8.

93. Bahrami A, Vafapour M, Moazzami B, Rezaei N. Hyperinflammatory shock related to COVID-19 in a patient presenting with multisystem inflammatory syndrome in children: first case from Iran. J Paediatr Child Health. 2021;57(6):922-5.

94. Carraro M, Rocha Rodrigues B, Rodrigues Jr. V. Case report: multisystem inflammatory syndrome in children (MIS-C) associated with SARS-CoV-2 with coronary involvement. Arch Clin Biomed Res. 2020;4(6):760-5.

95. Buosenso D, Di Sante G, Sali M; CURE COVID-19 Study Group. Cytokine profile in an adolescent with pediatric multisystem inflammatory syndrome temporally related to COVID-19. Pediatr Infect Dis J. 2020;39(8):e213-5.

96. Richardson KL, Jain A, Evans J, Uzun O. Giant coronary artery aneurysm as a feature of coronavirus-related inflammatory syndrome. BMJ Case Rep. 2021;14(7):e238740. 